$\mathrm{JD}$

78,1

60

Received 5 December 2020

Revised 27 April 2021

24 August 2021

Accepted 9 September 2021

\title{
Making time/breaking time: critical literacy and politics of time in data visualisation
}

\author{
Veronica Johansson \\ Swedish School of Library and Information Science (SSLIS), University of Borås, \\ Borås, Sweden and \\ Department of Science and Technology (ITN), Linköping University, \\ Linköping, Sweden, and \\ Jörgen Stenlund \\ School of Science and Technology, Örebro University, Örebro, Sweden and \\ Department of Science and Technology (ITN), Linköping University, \\ Linköping, Sweden and \\ Department of Behavioural Sciences and Learning (IBL), Linköping University, \\ Linköping, Sweden
}

\begin{abstract}
Purpose - Representations of time are commonly used to construct narratives in visualisations of data. However, since time is a value-laden concept, and no representation can provide a full, objective account of "temporal reality", they are also biased and political: reproducing and reinforcing certain views and values at the expense of alternative ones. This conceptual paper aims to explore expressions of temporal bias and politics in data visualisation, along with possibly mitigating user approaches and design strategies.

Design/methodology/approach - This study presents a theoretical framework rooted in a sociotechnical view of representations as biased and political, combined with perspectives from critical literacy, radical literacy and critical design. The framework provides a basis for discussion of various types and effects of temporal bias in visualisation. Empirical examples from previous research and public resources illustrate the arguments.

Findings - Four types of political effects of temporal bias in visualisations are presented, expressed as limitation of view, disregard of variation, oppression of social groups and misrepresentation of topic and suggest that appropriate critical and radical literacy approaches require users and designers to critique, contextualise, counter and cross beyond expressions of the same. Supporting critical design strategies involve the inclusion of multiple datasets and representations; broad access to flexible tools; and inclusive participation of marginalised groups. Originality/value - The paper draws attention to a vital, yet little researched problem of temporal representation in visualisations of data. It offers a pioneering bridging of critical literacy, radical literacy and critical design and emphasises mutual rather than contradictory interests of the empirical sciences and humanities. Keywords Bias, Critical design, Critical literacy, Data, Radical literacy, Representation, Temporality, Time, Visualisation
\end{abstract}

Paper type Conceptual paper

\section{Introduction}

It has been said that the beginning of the 21st century ushered in the third "golden age" of visualisation, this time uniquely signified by a skyrocketing usage of data visualisations in every

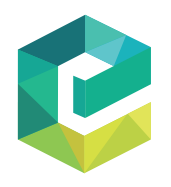

(C) Veronica Johansson and Jörgen Stenlund. Published by Emerald Publishing Limited. This article is published under the Creative Commons Attribution (CC BY 4.0) licence. Anyone may reproduce, distribute, translate and create derivative works of this article (for both commercial and non-commercial purposes), subject to full attribution to the original publication and authors. The full terms of this licence may be seen at http://creativecommons.org/licences/by/4.0/legalcode

The authors wish to thank the anonymous reviewers for insightful and valuable comments that helped strengthen the paper. 
conceivable context (Friendly, 2008a, b). Two decades later, we may confirm that the prevalence and perceived value of data visualisations remain and have increased: we find them in the news; in our personal smart devices; on company websites; as scraped feature snippets of web content appearing in connection with Google searches; and as compilations of our engagements with service providers from electricity to banking and grocery shopping, whether solicited or not. As delimited here, data visualisation refers to graphical displays or representations of data specifically designed to "... communicate precise information and values" (Beer and Burrows, 2013; c.f. also Kennedy et al., 2016), but their purposes include not only the communication of quantities but also (other) qualities of data and datasets. The same dataset can bear many potential meanings and form the basis for numerous different narratives.

The meanings or "aboutness" of datasets become realised and enacted in the data visualisations through visualisation designers' more or less conscious choices to focus on certain data variables and giving them particular graphical representations with relative weight and prominence in the overall visual design. This can be, for example, as when data on votes in the US election in 2020 become visualised with emphasis on geographic distribution by colour coding each state in accordance with the winning candidate. Alternatively, by shifting the representational focus, this same data can also be graphically displayed so as to communicate the overall distribution of votes between income classes. In this article, we focus on yet another category of representational features in visualisations of data - the graphical display of time and temporality.

For the purpose of the article, we present a theoretical framework rooted in a sociotechnical view of representations as biased and political, combined with perspectives from critical literacy, radical literacy and critical design. The framework provides a basis for discussion of various types and effects of temporal bias in visualisation. Empirical examples from previous research and public resources illustrate the arguments. Thereafter, we present a four-dimensional model that suggests that appropriate, critical, user approaches and design strategies require various dimensions and combinations of critical and radical literacy, and how these can be supported through conscious critical design strategies.

\section{Bias and politics of temporal representation}

We rely in the following on a sociotechnical view of representations as biased and political. In its broadest sense, bias denotes a disproportionate inclination for or against someone or something. A further connotation associates such unbalanced or partial perspectives with unfair, prejudiced or harmful views and effects. Although bias is commonly associated with human mental processes (c.f. e.g. Dimara et al., 2020), corresponding discussions prevail also in relation to information systems, for example, in algorithm construction and operation (e.g. Johnson, 2020a, b). Such understandings of bias, as described by Johnson, acknowledge them as ubiquitous social patterns, mutually shaped in interactions between "the social" and "the technological". Viewing representations in visualisations of data as sociotechnical constructs we start, thus, from the view that they are inevitably biased. No representation can provide a full, objective account of "reality" (or in this case, "temporal reality") but offer instead partial and/or subjective viewpoints.

The corresponding connotation of politics here falls back on Leftwich's definition of politics as integrated, everyday relations of power and distributions of resources. The concept of resources includes all that holds value, from land and property to "... time, education, status, influence, health and knowledge" (Leftwich, 1983, p. 12). Politics too, in this sense, is seen as continuously produced and reproduced in human activities involving and relying on technology; shaped by and shaping institutions, culture and ideology on all levels of scale (Leftwich, 1983, p. 11). The connection between representational bias and politics, in turn, relates to the view of representations as inseparable from human, social, meaningmaking and related actions. Latour and Woolgar, for example, describe how cultural conventions and practical considerations become reproduced in new representations, each 
JD

78,1

62

time repeating and reinforcing values, ideas and unreflected habits among their producers and users (Latour and Woolgar, 1986/1979). In the words of Hutchins (1995, p. 82), representations exert disciplinary power over the ways users reason, think, act and solve problems; they support certain ways of thinking and acting and hide or suppress others.

In many cases, the choice to include or adapt a certain representation in a specific context can be explained by reference to dominant, socially agreed upon cultural norms and traditions (c.f. Blackwell, 2011), which means that their uses are associated with comparatively conservative tradition and unreflected reproduction. Representations of time and temporality are no exceptions. Insistent critique maintains that dominant temporal representations used in an empirical science paradigm or framework might give rise to the misunderstanding of time as associated with rectilinear progress. Combined with the idea of human rationality this might result in various detrimental consequences. The philosopher Sampson, for example, warns, with mechanical clocks as example, that although useful for structuring, managing and controlling the world, they also contribute a false conception of what we can reach and understand and how (Sampson, 2020, pp. 8-9). As alternative and opposition to the "Kantian" linear and arrow-like idea of time as uniform, unidirectional, straight and infinite, the philosopher Critchley proposes a conception of "times" in the plural as a spiral or series of loops, ". . reversible, intermittent, episodic, various and variable, pluriform, relative, relational, and, importantly, finite" (Critchley, 2016). And visual theorist and digital humanities scholar Drucker advocates humanities adapted supplements to what she perceives as the dominant logics of "empirical science" temporalities, since "... in the humanities time is frequently understood and represented as discontinuous, multi-directional, and variable" (Drucker, 2014, p. 75).

Indeed, the majority of temporal representations in data visualisation invoke and communicate variations of precisely such linear notions of time critiqued above. Ayalasomayajula (2016) identifies seven main temporal representations in visualisation in terms of: line graphs, stacked area charts, bar charts, Gantt charts, stream graphs, heat maps and polar area diagrams. The first five mainly rely on linear representations of time and display events or entities as connected to singular instants in time or to specifically delimited temporal intervals (c.f. Aigner et al., 2011). The last two, however, the heat map and the polar area chart, can in more direct and primary manners illustrate the occurrence of events and entities as cyclical, temporal patterns (on, for example, daily, weekly or yearly bases).

Another aspect to consider is the choice of temporal resolution (i.e. the scale or granularity of the time) which sometimes is crucial. Enabling users to compare important interrelated processes occurring on different time scales can be critical, for example, while communicating information regarding present-day environmental issues such as climate change. A relevant example in this context would be to compare the amount of carbon dioxide emitted to the atmosphere during the last 100 years, due to consumption of coal, with the time required for ancient plants to withdraw the same amounts of carbon dioxide via photosynthesis from the atmosphere to enable the formation of the coal in the first place. Comparisons of this kind, comprising a concrete perceptible time frame and an abstract imperceptible long time frame rooted in deep geological history are less frequent and require careful design choices. Designers of data visualisations will thus continuously make more or less conscious choices between temporal representations, but irrespective of choice, in light of the theoretical perspectives advanced here, the process itself is an exercise that contributes to the production and distribution of values in society.

In contrast to sociotechnical perspectives, the majority of visualisation research and design to date, however, tends instead to focus on and approach various forms of bias (temporal and otherwise) as largely external to the visual representations themselves from one out of two perspectives: the first takes hold of biases as originating from pre-existing cognitive biases among users (c.f. e.g. Dimara et al., 2020); the other focusses on bias in the data as originating from data collection and procurement processes (e.g. Johnson and 
Sanderson, 2003; Bonneau et al., 2014, pp. 10-11; Gschwandtner et al., 2016; Levontin et al., 2020). In both approaches, seeing these issues as predating and external to the visualisation process, its products and user interactions sets the foundation for the common assumption that problems of bias can be identified and corrected or at least balanced, through conscious design strategies. But without acknowledgement that visualisation itself also repeats and perpetuates old, or produces new, biases with political consequences, problems of bias cannot be fully addressed. Although a number of empirical studies illustrate resulting political consequences through the construction and use of data visualisations in various contexts (e.g. Beaulieu, 2002; Joyce, 2005; Lurie and Mason, 2007; Johansson, 2012; Bendoly, 2016), critical approaches of reflexive character are still rare in the field. To incorporate such views, we turn to a further set of theories and methodology from critical literacy, radical literacy and critical design.

\section{Critical literacy, radical literacy and critical design}

The widespread subjections to and uses of data visualisations among non-experts (groups and individual members of society) are accompanied by relative agreement among researchers that users need certain competencies - "literacy skills" - to adapt to and make proper use of them. The skills involve competencies enabling individuals to access, read, write, interpret, manage and transform information in various modes (i.e. verbally and/or non-verbally coded) and modalities ("senses" i.e. auditory and/or visual) (Moreno and Mayer, 2007). The associated literacy approaches are likewise mode and modality-specific, as expressed in for example "numerical literacy", or "numeracy" (e.g. Craig and Guzmán, 2018), "data literacy" (e.g. Calzada Prado and Marzal, 2013; Mandinach and Gummer, 2013; Koltay, 2015) and "visual literacy" (e.g. Elkins, 2009; Brumberger, 2019).

Even though these literacy approaches often include some sort of critical perspective or dimension, they fall short of the interest here for three reasons. Firstly, each of them primarily focusses on one mode and/or modality in particular. Even multimodal literacy (c.f. Jewitt and Kress, 2003) can be said to rely on modal separation of meaningful qualities of communication. Representations of time, on the other hand, play out across modes and modalities in visualisations of data. Secondly, these approaches are also frequently developed for instructional and pedagogical learning in formal contexts, whereas biased representations with political consequences permeate the social fabric to an extent that is difficult if not impossible to handle in institutionalised teaching and learning alone. Thirdly, the critical perspectives in most of these are not developed enough to meet the challenges posited by the theoretical framing of the problem complex here. What is required are encompassing, comprehensively critical approaches, with application potential outside of formal teaching. For this purpose, we position this article in the traditions of critical and radical literacy, with an extension to ideas in critical design.

Critical literacy is an approach and methodology with strong connections to the Frankfurt school, (Neo-)Marxism and critical theory in the 1920s, and the Brazilian educator Paolo Freire's theories on critical pedagogy from the 1940s onwards. It is grounded in the view that communication, irrespective of form and technology, mediates - beyond simplified notions of "content" - discriminatory or prejudiced worldviews and knowledge claims (Lankshear and McLaren, 1993). Critical literacy in this sense is primarily associated with pedagogical application in language and literature classes, aiming for liberatory, revealing processes of critical analysis through dialogic reading and similar strategies that help uncover underlying, embedded, and difficult to identify meanings of - primarily - texts (Lankshear and McLaren, 1993).

In the early 2000s, these critical literacy ideas started to break through in library and information science (LIS) as well. Tewell (2015) describes as a "seminal" publication Kapitzke's (2003a; c.f. also Kapitzke, 2003b) poststructuralist critique of traditional 
JD

78,1

information literacy, advancing the critical information literacy expansion in the field towards engagement with similar "... social and political ideologies embedded within the economies of ideas and information" (Tewell, 2015, p. 29). Researchers in the ensuing tradition emphasise in various forms of critically oriented information literacy approaches associated with competencies, not as tools for learning "proper" uses of information systems and "content" of information resources, but as tools for questioning the knowledge claims made and preserved through the same (c.f. also e.g. Tuominen et al., 2005; Andersen, 2006). Many of these approaches, however, have come to "halt" the critical agenda with a comparatively passive conception of information users restricted to questioning, critiquing and selecting among existing resources of the surrounding information landscape, as argued, e.g. in Johansson and Limberg (2017). Freire's critical pedagogy, on the other hand, propagates also an active agency for the transformation of the status quo.

In line with these most far-reaching critical aspirations of Freire's ideas, we emphasise here that critical literacy comprises not only evaluative but also transformative aspects (c.f. Johansson, 2012; Johansson and Limberg, 2017). Ultimately, the aim is to support the generation of new, alternative, forms of knowledge and information, in favour of diversity, minorities and the otherwise marginalised and oppressed. It stresses the importance of influence over and access to diverse, flexible knowledge resources to enable users and learners to act in their personal and their communities' interests (Kapitzke, 2003a, b; Johansson, 2012). This, in turn, includes opportunities for active involvement of marginalised groups in changing systems, technologies and associated processes for information construction, representation and use. The way we align with and apply critical literacy here is therefore also in line with various radical literacy approaches in LIS.

Radical approaches within LIS have, for example, been applied in community-based, transformative library pedagogy (Riedler and Eryaman, 2010) and for developing public libraries into spaces for democratic empowerment (Eryaman, 2010). Closest to our interests and aims here, however, are Whitworth's ideas on radical literacy (Whitworth, 2014). Radical literacy, in Whitworth's political and transformational agenda, takes a broad approach to communication and knowledge-formation outside of formal educational contexts, including all of the ubiquitous and perpetual information and knowledge processes of everyday life; across technology, platforms and modes. The methodology offers cognitive tools through which we may “. . . see the potential for transforming systems of informational exchange into more democratic forms, and thus more widely distributing cognitive authority" (p. 6).

Similar to Whitworth's socially pervasive radical literacy approach, we too go beyond delimitations to formalised education to discuss in a broader sense the implications of design choices for representation, meaning-making and related actions in everyday social interactions, and ask, as an extension of this, whether critical/radical user approaches in these contexts can be supported through conscious design strategies. The correlation between critical pedagogy and critical theory in the realm of information and information systems design is critical design, first introduced by Dunne and Raby (2001). They posit that all design inevitably falls into one out of two categories: affirmative or critical. The first perpetuates the status quo, repeats and reinforces such representational biases and politics discussed above. The second takes as an ethical obligation the intention to design in ways that strive to make bias visible and afford the empowerment of users (Lankshear and McLaren, 1993, p. 58). Criteria of critical design include the affordance of perspectivechanging accounts of a given phenomenon; a problematising ("speculative") rather than declarative ("truth-claiming") approach; a reflection of dialogical methodology; the improvement of cultural competence on behalf of users; and reflexive awareness of the design process itself as an actor (Bardzell and Bardzell, 2013). 


\section{Four types of temporal politics}

The theoretical framework presented above delineates the view of bias and politics of (temporal) representations as a problem of repeating and possibly reinforcing undue inclinations for or against someone or something in unreflected and/or prejudiced and harmful ways, so as to influence the social distribution of values. This raises several urgent questions for data visualisation, the first set of which are addressed in this section and concern why certain representational choices are made; who has the power to decide and influence the choice and variety of these; and what consequences that may follow. The focus helps identify challenges to and needs of critical and radical user approaches and design strategies.

The argument in the following is structured around four types of temporal bias in data visualisations and possible associated political effects. For each type, we discuss what aspects should be critically questioned and why. The examples are separated for the sake of clarity of argument and should not be understood as mutually exclusive - on the contrary, they are more than likely to overlap in practice.

\section{Limitation of view}

The first examples of political effects due to temporal bias presented in this article are primarily connected to unreflected reproduction of traditional, "customary" - and thereby powerfully normative by convention - ways of representing data with respect to time, which leads to limitations of view on behalf of both users and designers. Representational conventions develop and solidify on a societal level as sociocultural norms and traditions (e.g. Blackwell, 2011; Drucker, 2014). They can also take the form of more local cultures and traditions within and between specific organisations and professional domains (Johansson, 2012; Bendoly, 2016). As visual representations are both biased and biasing, simultaneously constructed upon and inferring possibly severely restricting disciplinary power (c.f. Hutchins, 1995), they facilitate and enact mutually shaping and reinforcing effects between institutionalised conventions of representation and users' interactions with and appropriations of them. The (traditional) choice of representations affects what questions that become asked, what knowledge is deemed important and what representations are favoured in future representations. These social processes are large-scale, naturalise over time and make it increasingly difficult for both users and designers to identify and/or retain awareness of inherent limitations in particular representations for the data and purposes in question.

Taking traffic accidents as example, the three visualisations in Figure 1 illustrate two of the most common temporal representations of such data (1A and $1 \mathrm{~B})$ and one more unusual (1C). Figure $1 \mathrm{~A}$ presents a screenshot of an interactive visualisation of Transport for London (TFL) accident data for the year 2015 (Kocinkova and Hale, n.d.). The line diagram in 1B visualises the number of fatal traffic accidents in the EU over the course of 10 years (Eurostat, 2020). Like the TFL example (1A), the Eurostat visualisation (1B) uses an aggregated temporal representation of accidents per year but also adds a longitudinal, developmental perspective by placing aggregated numbers for a succession of years alongside each other. Concurrent with strong organisational and professional traditions in traffic-related work (c.f. Johansson, 2012), visualisation 1A is primarily geographical, emphasising where over when, whereas $1 \mathrm{~B}$ is a direct example of the "Kantian, linear, temporal representation" critiqued by humanities scholars (Drucker, 2014; Critchley, 2016; Sampson, 2020). Both displays are conformant with tradition and powerful in their own rights and contexts of use, yet also biased and political as both offer powerful and convincing yet restricted, partial and valueladen temporal analytical and narrative views.

The case in point can be illustrated by a study at a municipal office that used a visualisation of traffic accident data similar to example $1 \mathrm{~A}$ to analyse patterns of traffic
Making time/ breaking time 
$\mathrm{JD}$
78,1

66

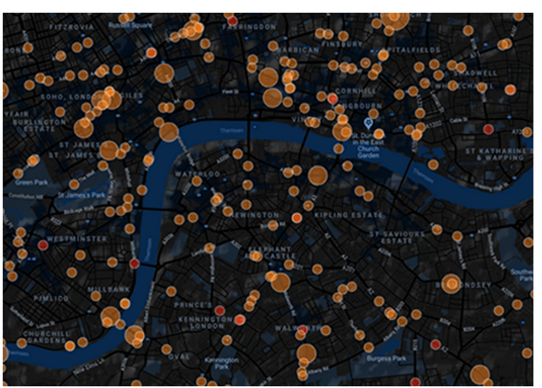

(A)

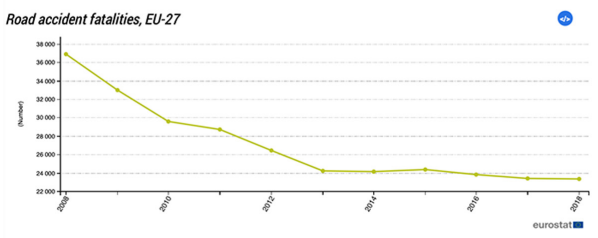

(B)

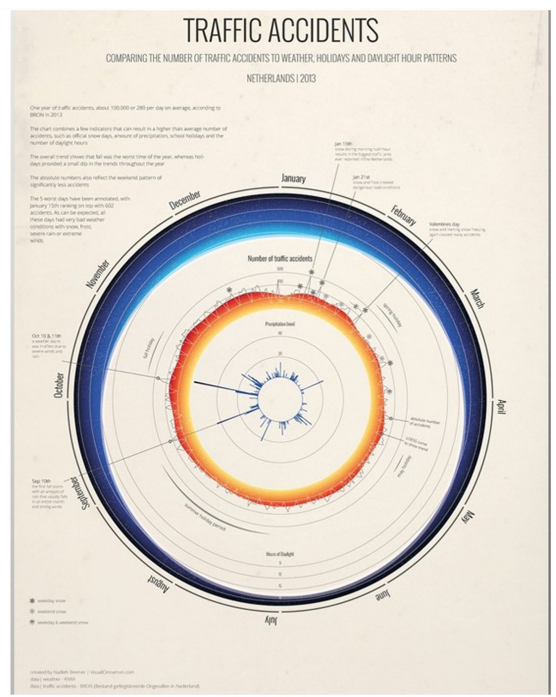

(C)

accidents in the city for purposes of reducing accidents and fatality (Johansson, 2012). This data had always been primarily geographically visualised, from analogue practices of placing pins in maps on the walls of the office, to subsequent digital geographic information systems (GIS). Following the disciplinary power (Johansson, 2012) of the visualisation, the analysts focussed on geographic patterns of accidents per year, addressing them with geographically related measures such as setting up traffic lights, clearing vegetation and adjusting speed limits at specific high-risk places. With digitisation and intensified efforts, measures were increasingly successful, but it was not until most or even all of the geographically related causes of accidents had been eliminated, that the limitations in the visualisation became more obvious. Once most of the geographically related causes had been rectified, accidents still occurred but without displaying meaningful patterns in the geographically based visualisation. Turning to the longitudinal yearly measurement of numbers in the $1 \mathrm{~B}$ example, and in relation to traffic accident data in specific, yearly quantitative figures and measurements are closely connected to political tools and interests in terms of goals and evaluation. Relying on the temporal representation of this type might therefore reflect and reinforce the interests and needs of formal political actors and agendas, introducing and enforcing time as a powerful instrument dictating the distribution and use of resources, whilst simultaneously likewise obscuring or hiding potential factors of relevance for practical analytical and counter-measure work aiming to better understand and ultimately reduce causes and occurrences of traffic accidents (c.f. e.g. Johansson, 2012).

Each singular representation in Figure $1 \mathrm{~A}$ and $1 \mathrm{~B}$ can thereby be seen to be at risk of limiting - "disciplining"-(Hutchins, 1995) the user's view with associated detrimental effects. The dominant spatial framing of $1 \mathrm{~A}$ introduces the risk of disciplining subsequent analysis and interpretation towards a geographical bias, and the compression of data into an aggregated value for a whole year in both $1 \mathrm{~A}$ and $1 \mathrm{~B}$ hides potential temporal dynamics, variations, shifts, correlations and patterns on smaller, larger or other temporal scales (c.f. Aigner et al., 2011). However, a mere juxtaposition of representational alternatives for the same or similar datasets as accomplished by the side-by-side presentation of examples in 
Figure 1 highlights to a user that no singular representation is the only "right" or "possible", but that there is a range of alternative time-related variables that may be of significance for representing, analysing and interpreting data. A similar critical effect can also be achieved within a stand-alone visualisation by incorporating innovative and/or multiple temporal representations, as in $1 \mathrm{C}$.

Bremer's (2015) visualisation of traffic accident data in another context (Figure 1C) also refers to the calendar year but then deviates from previous examples in numerous ways. The example offers a rich, multidimensional "visualisation wheel" that also represents correlations of accidents with several other temporal variables of both cyclical and recurrent (such as seasons, school holidays and daylight hours) and unique (weather conditions) variables.

Considered together, the examples in this first category illustrate the limiting powers of representational conventions but also ways in which multiplicity and alternation of temporal representations provide perspectives and accentuate the multidimensional temporal nature of, in this case, traffic accidents. Allowing possibilities for users to see and maybe by their own accord shift and move data between different representations with multiple granularities (different time scales; hours, days, weeks, months and years) and other temporal variables (seasons, holidays and weather) offers potentials for qualitative exploration of variables relating to social and individual arrangements and activities in many temporal dimensions and with numerous potential bifurcations.

\section{Disregard of variation}

Our second set of examples of politics connected to temporal bias include visualisations that treat a certain temporal representation as universally applicable across contexts and users, assuming a more or less conscious view of this representation as generically valid. However, few social and individual actions and phenomena are so unaffected by other internal or external factors that they will measure and compare equally, fairly and representatively against a standardised, mean, average or similar temporal representation presuming universal applicability. On the contrary, using temporal indicators as yardsticks for values may easily lead to cases in which a purportedly "objective" time measure unjustly and harmfully misrepresents, discriminates or excludes an individual person or collective entity (an organisation, a group and a nation). Time is not a universal, constant, objective entity that can justly measure and compare social values without contextualisation, qualification and/or adaptation. Even in those cases where the application of a standardised temporal representation is, on the whole, warranted and legitimate, related practices and consequences should still be carefully evaluated.

Particularly misconstrued applications of visualisations that measure personal or social data against standard, generically framed values (including of temporal character) have been noted not least within the so-called "quantified self" movement and personal health data surveillance applications. van Dijk et al. (2016) describe one example in which temporal "standards" or median and mean values for larger groups set up as authoritative indicators of what should be considered good, bad or risky values - in this case concerning heart beats per minute - can deviate so much from meaningful values for individuals that they risk severe misjudgment of oneself and others.

Purposive or arbitrary "trimming" of the timeline might result in similar political consequences. Consider, for example, a visualisation of an individual's credit score (creditworthiness) based on personal financial data over the standardised time frame offered by a fictive credit company as illustrated in Figure 2A (similar to many existing application-based services with data visualisation features). The standard time frame allowed in common services of this type tends to be comparatively short. Even though a user 
JD

78,1

68

in this scenario can choose between calculating and displaying a credit value based on personal transactions and financial state over time periods ranging from the last month to the last year, it is still a comparatively short time span. Sudden, unexpected or extraordinary events during this interval may easily result in a non-representative or comparatively "unfair" valuation of an individual. A longer time frame could have illustrated this, or "evened out" a brief financial imbalance or "dip", but this potential is hindered and hidden from view in a design such as that in the example, and there is no way that the user him-/herself can affect the analysis and display of values to remedy the imbalance (bias) in visualisation services of this kind.

The example in Figure 2B, on the other hand, illustrates instead the usefulness of locally adapted data visualisation for local community needs, in this case for self-organised governance, control and coordination of actors and actions in time. The visualisation is a simple heatmap illustration of customer frequency per weekday and hour at a local supermarket in a small Swedish town. This visualisation was shared with the supermarket's customers via social media as a tool for them to self-adjust their chosen times for grocery shopping in order to support national and community level requests for social distancing during the COVID-19 pandemic. The store in question is part of a large national chain, but interestingly, the chain made no attempt to produce a "general" national visualisation based on aggregated visitor data from all the stores. Instead, the chain's local stores produced local heatmaps, which suggest a more sophisticated understanding that not only opening hours may differ between stores, but that the temporal patterns for customers' shopping activities may also display significant local variation.

The measurement and comparison of individual persons and collective entities against generalised, "generic" temporal standards, perhaps of arbitrarily chosen or purposefully trimmed or manipulated time spans, might cause great differences in the outcome of deductions and decisions of a deeply political nature. If combined with a lack of data or other explanatory resources that may contribute with other factors of relevance in individual cases, the risk of negative political consequences due to the restricted temporal representation increases. And the stakes can be high: who, for example, will get a loan, a good grade or proper attention by a physician? How useful will the information displayed in the visualisation be for the data subjects themselves? The $2 \mathrm{~B}$ example also implies that more

Figure 2.

(A) Temporal representation of an individual's creditworthiness. Fictive example, (B) ICA Toria Supermarket (2020), Reproduced with permission

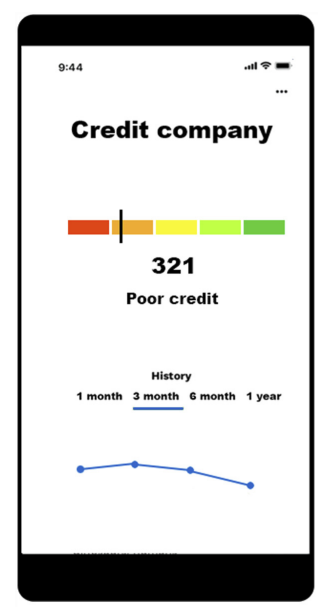

(A)

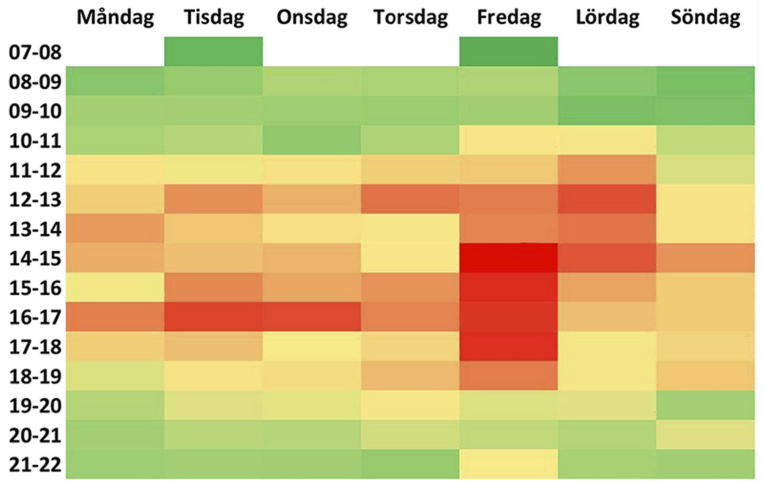

(B) 
sophisticated responses require not only an understanding of potential bias in generic representations but access to data and tools that allow flexible, local and/or personal adaptations or adjustments.

\section{Oppression of social groups}

The third type of political effects from temporal bias comprises examples in which temporal representations can be seen as expressions of pre-existing structural inequalities through which the rules, values, interests and perspectives of dominant social groups overshadow and suppress corresponding ones of less powerful groups. This differs from the previous examples of temporal bias in the sense that "undue inclinations" in this category are more readily associated with long-standing social inequalities affecting the distribution of power and resources between identifiable social groups. Typically, these social groups are described in terms of race, gender and social class, and sociotechnical research is paying increasing attention to how science and technology "... contribute to or enable resistance to enduring inequalities ..." structured around such categories (Hess et al., 2017, p. 319). For this type of bias, it is more difficult to find "worse" and "better" real-world examples of oppressive, "hegemonic", representations in the digitised definition and delimitation of visualisations that we have made here since the oppression itself continuously excludes the marginalised from representation in both (data) contents and design processes of relevant temporal visualisations. This state of affairs underscores the importance and urgency of the discussion and analogue examples from critical movements within spatial and geographical representation and (data) visualisation provide further testimony to critical and radical literacy challenges and potentials in the area.

To a certain extent, a broadened, "democratised" access to existing data resources and visualisation tools can go some way to support the political interests of marginalised groups against more powerful actors. There are many examples of local community empowerment through the use of data visualisation, although most commonly relying on GIS for surveillance of land resources. Similar examples of empowerment of marginalised groups through data visualisation that mainly relies on temporal representation do exist but seems less common. One such example is provided by Hsu et al. (2017), who describe a case including visualisations of data on air quality and pollution over an extended time period, for the political purpose of supporting local community interests against more powerful actors. Another structurally oppressive representational dominance is described by McKenzie et al. (2017), highlighting mechanisms that exclude Indigenous temporal representations due to the dominance of ("western" or "Global North") cultural and scientific expressions of linear time scales depicting a steady, linear progression from past to future with standardised and regularised sets of granularities (days, hours, minutes, etc.). What is lost from view are alternative time perceptions in Indigenous cultures in terms of localised perceptions, combining, for example: “. . . multiple senses of linear time and a variety of cycles that give a qualitative sense of whether a moment is a good time or not for an activity" (McKenzie et al., 2017, p. 46). This marginalisation of alternative representations of time may not only be a loss for the local knowledge holders themselves but a loss of plurality of views, voices and perspectives for society at large.

Through the acknowledgement of structural oppression by means of geographic representations (maps and cartography), radical approaches are facilitated by inviting and supporting marginalised groups to produce their own, alternative representations. One such example, incorporated below, comes from the "This Is Not an Atlas" project, illustrating transformed maps as part of political struggle (notanatlas.org, n.d.). Figure 3A shows the alternative way (compared to the official cartographic representation of the same geographical area) that an Indigenous community in the Philippines visually represents
Making time/ breaking time 
$\mathrm{JD}_{78,1}$

70

Figure 3.

(A) Mapa da Aldeiaboa vista (n.d.), CC BY-NCND 4.0, (B) Nygårds (2020), Reproduced with permission

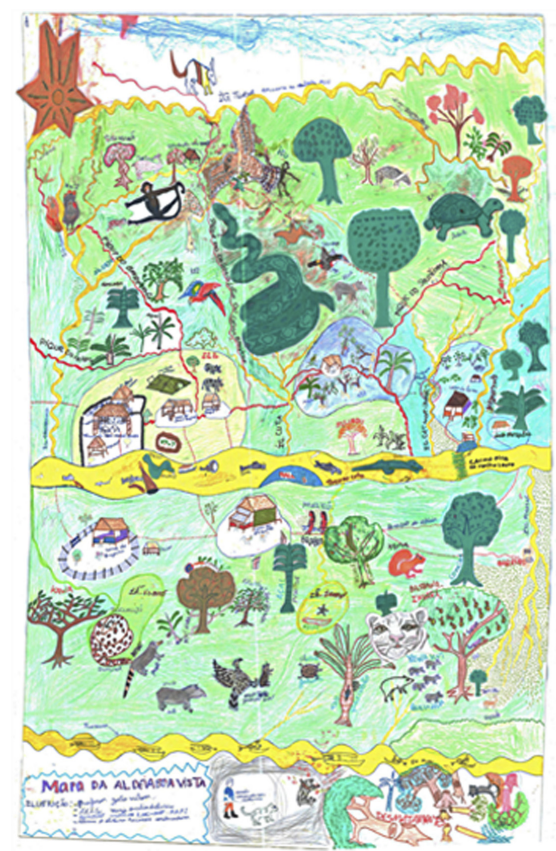

(A)
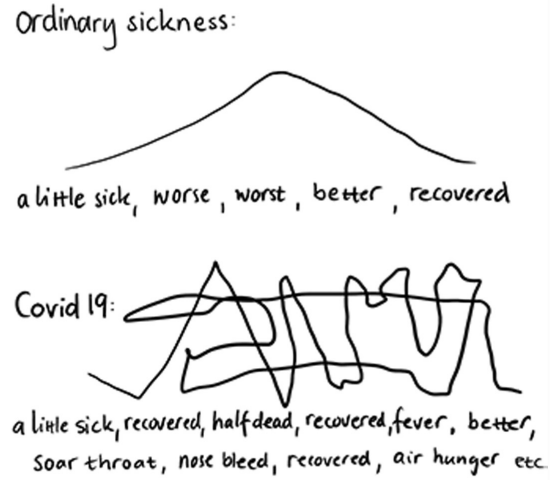

TOTALLY UNPREDICTABLE

their territory. Corresponding transformed or alternative representations of temporally structured data visualisations produced by disempowered or marginalised groups and individuals seem less common. The example in Figure 3B demonstrates, however, that similar transformations of temporal representations are both needed and possible. The image shows a partly humoristic, partly critical rendition of an individual's experience of suffering from COVID-19, an experience that did not measure well onto the medical system's standard temporal description of a typical virus infection progression (Nygårds, 2020). The drawing illustrates first the medically established "normal distribution" time curve for the progression of an infection, deployed and assumed by medicals, a professional group with high status and power over individuals in need of care. This is then contrasted with the individual patient's own representation of her non-conformant experience of the progression of the sickness.

It should be the prerogative of the oppressed, marginalised and misrepresented to identify, challenge and oppose the instruments of oppression and their effects. Unfortunately, this is not easily accomplished - whether in data visualisation or otherwise - as part of the problem is precisely the lack of power and resources among these groups to even be part of a dialogue. Taking as broad perspectives into account as possible, an elaborated cultural diversity in the representation of time is highly warranted as argued from the perspective of Indigenous cultures by Mackenzie et al. (2017). However, whereas counter-mapping is well established, there is no corresponding "counter-timing" research field or grassroot movement in the area of temporal regimes. Attempts and examples may be found but are more like isolated occurrences than parts of an established counter-movement as we see in the realm of geographical representations. The absence of such an established "critical temporality" approach is all the more lamentable, since the politics at stake is equally high, and there is nothing to suggest a weaker potential of similar endeavours. 
Finally, it should also be noted that this concern and aim can and should be extended to structural inequalities affecting social groups not merely between grand constructions of East/South and North/West, but within contemporary societies wherever their global positions and cultural belongings may be. Increasing reliance on data, visualisation and even automation and AI is likely to increase structural inequality in terms of uneven access to resources such as power, knowledge and information concerning how and why data is analysed and presented. This, in turn, as the sociotechnical perspective emphasises, shapes what knowledge is privileged and what actions and decisions are made and the consequences for individuals and groups in all contexts can be considerable.

\section{Misrepresentation of topic}

The fourth and final type of political effects of temporal bias presented here concerns deficiencies or inadequacies in existing temporal representations for adequately conveying desired narratives. These types of problems can be seen to arise in situations where visualisations are intended to represent temporal entities or processes that are somehow "above", "beyond" or unparalleled in conventional representational repertoires of time and temporality. Attempting, in lack of alternatives, to "shoehorn" a dataset into a temporal representation that is not fit to represent necessary and critical aspects of the same causes bias in that erroneous or misleading narratives are emphasised, with potentially serious political consequences.

One variety of this problem can occur if some of the design aspects of a visual representation compete with each other or even become contradictory. For example, the narrative of a dynamic visual representation can be impaired by an uneven distribution of events in the underlying dataset. This is often the case in historical accounts since usually much more is known about the recent past compared to the more distant past. This might inspire designers to alter the representation of the passage of time (i.e. the time rate) in an animation to prolong shorter and eventful time intervals and shorten longer time intervals with relatively few events. However, Lee et al. (2011) caution the use of visualisations that alter time rate in animations since such time "distortions" make temporal aspects even harder for the user to interpret. It also accentuates tendencies to overestimate very short time periods and underestimate very long time periods. Another example is provided in a study by Stenlund and Tibell (2019) in which the scenario of human evolution from our earliest bipedal ancestors during the last seven million years was represented in a spatio-temporal dynamic visualisation, produced in four versions with a different temporal representation in each. The results show that different aspects of the depicted evolutionary scenario became emphasised depending on which temporal representation was used. For example, when the time rate was the same throughout the visualisation, the very short fraction of time that modern humans have existed (only $4 \%$ of the depicted time frame) became very apparent to the study participants. The choice of temporal representation may thus have far-reaching political implications for how users interpret data visualisations regarding issues such as the rate of current climate change and loss of biological diversity due to human activities.

Lemke (2000) refers to the usually weak interrelationships between events and processes occurring at different temporal scales as the adiabatic principle but at the same time acknowledges what he calls heterochrony, unique occurrences when long-timescale processes cause significant changes in much shorter timescales. Tipping points is an example of this kind of occurrence. The possibility that the Earth is approaching a so-called climate "tipping point" is another such issue that has proven difficult to visualise in comprehensible ways. As an example, we illustrate with two visualisations from the not-for-profit environmental lobbying and educational organisation Job One for Humanity. They present several visualisations to argue for the critical state of the Earth's environment and the "four most 
$\mathrm{JD}$

78,1

72

critical tipping points" (Job One, n.d.a, b). One of these "tipping point" visualisations correlates $\mathrm{CO}_{2}$ emissions with global warming (Figure 4A). In the long temporal perspective, the dramatic exponential increase of both values after the industrial revolution makes a powerful argument. But there is of course no data that can illustrate the tipping point itself, and what would happen once we pass it. In attempting to illustrate a break in evolutionary time of the magnitude imagined, the organisation therefore turns to an infographic (Figure 4B), a visualisation of a "freer" graphic character than a data visualisation which visualises precise (real) values (c.f. Beer and Burrows, 2013).

Attempting to communicate temporal relationships such as the examples mentioned above through data visualisation is challenging since it requires careful consideration on behalf of the designer as to how different scales can be visually combined without distorting the user's perception of time in directly false, unintended or otherwise misleading ways (Stenlund, 2019). If the depicted processes are complex (e.g. climate change) or unfamiliar (e.g. "tipping points"), design compromises might be needed to communicate the intended information in a successful way. These may include scaffolding resources such as help texts or the decision to choose another means of communication such as the freer form of an infographic.

Figure 4.

(A) Job One for Humanity (n.d.a.), CCBY-NC, (B) Job One for Humanity (n.d.b.), CCBY-NC

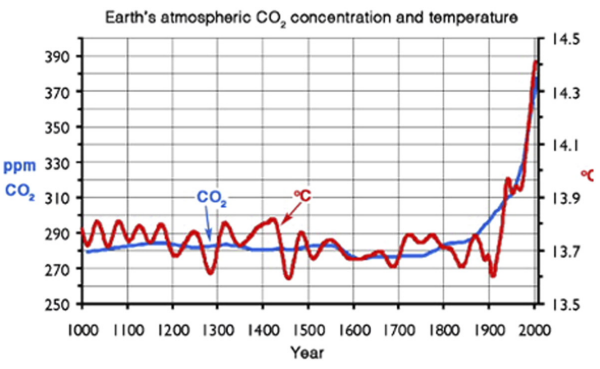

(A)

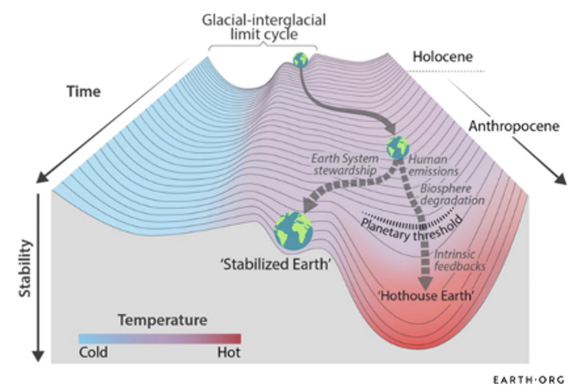

(B)

\section{Four dimensions of critical/radical literacy and design}

The preceding discussion presented four possible political effects of temporal bias. From the theoretical focus and interest here, a second urgent set of questions in relation to this concerns what users and designers of data visualisations can or should do in order to act critically "appropriate" in this difficult terrain; that is, so as to minimise or make such bias visible and afford the empowerment of users and social groups. Accordingly, we proceed by laying forth a corresponding conceptual model with four interrelated dimensions of critical and radical literacy. The model can be summarised with four "C:s", in terms of Critiquing, Contextualising, Countering and Crossing. We suggest that this model may serve as a conceptual lens through which to critically explore and explain new, invisible or taken-for-granted ways of representing, interacting with and thinking about, designing, using, teaching and valuing aspects and dimensions of time and temporality in visualisations. Each dimension is presented below, with references to relevant critical and radical literacy theory, visualisation examples from the preceding section and connections to related critical design strategies.

\section{Critique}

The first dimension places emphasis on the need to Critique and question representations of time and temporality; to "see" and "see through" representational bias and consider the 
appropriateness of temporal representation in the first place, along with alternative temporal scales, granularities or perspectives.

Limiting and biasing powers of the representational convention are at work on all levels and in all spheres of society. After a while, representations become natural, taken for granted as the proper, "true" or only way to represent the phenomenon in question; it becomes "blackboxed" (c.f. Latour and Woolgar, 1986/1979). Even users that are well "literate" in the traditional senses, may, because of this naturalisation and black-boxing, find it difficult to question a certain convention for temporal representation, reflect on and problematise its limitations and see other potential views beyond what the dominant display encourages or dictates (their "disciplinary power", Hutchins, 1995). The critical literacy challenge encompasses both individual visualisation critique ("what might be detrimentally restricting about the visual representation in visualisation X?"), as well as questioning the representational convention in a certain context, for specific issues or phenomena, in general ("is "accidents per year" really the most efficient visualisation strategy for traffic accident data?”) (c.f. also Tuominen et al., 2005; Andersen, 2006). It includes, in other words, a critical, questioning approach - critique - towards any and all instances of temporal representations for "undue" or "disproportionate" inclination or emphasis due to unreflective reproduction.

The examples previously provided in Figure $1 \mathrm{~A}-\mathrm{C}$ illustrate the ways in which multiplicity and alternation of temporal representations provide perspectives and accentuate the multidimensional temporal nature of, in this case, traffic accidents. Allowing possibilities for users to see and maybe by their own accord shift and move data between different representations with multiple granularities (different time scales; hours, days, weeks, months and years) and other temporal variables (seasons, holidays and weather) offer potentials for qualitative exploration of variables relating to social and individual arrangements and activities in many temporal dimensions and with numerous potential bifurcations.

In this particular context, established visualisation design strategies are well in line with other theories on how to destabilise and denaturalise the very foundations for temporal (and other likewise biased forms of) representation. It concerns ways to make "the invisible" or "naturalised" and "taken for granted" visible again. This type of limiting representational bias is also the most well-acknowledged in visualisation design theories and heuristics. A well-established mitigating remedy for making users aware, or reminding them, that a particular temporal representation is not natural and self-evident, nor the only possible, is primarily recommended to be solved by offering multiple representations (e.g. Ainsworth, 2008; Johansson, 2012). Already established strategies in terms of providing multiple representations provide possibilities to remind the user to critique the representation and remember that it is a human construct, by necessity biased and limited. We add to this also the suggestion that supporting different datasets for the same representation can work in similar ways as argued by, for example, Johansson (2012). As such, design choices to this effect are well in line with ideas and aspirations of critical design, which encourage, for the same purposes, the affordance of perspective-changing accounts of given phenomena and reflection of dialogical methodology (Bardzell and Bardzell, 2013).

\section{Contextualise}

The second dimension accentuates the needs and values of being able to Contextualise generic, standardised and externally defined values and measurements associated with time and temporality; to be able to adapt and reshape temporal data, values, scales and granularities, so as to suit and represent the needs and characteristics of the local and personal.

Basic knowledge of how and why externally provided standard temporal values along with omissions and purposive or coincidental "trimming" or other adjustments can cause 
JD

78,1

unjust representations and consequences are necessary for the user to at all respond critically. But allowing and assisting "the oppressed”, in Freire's (2000/1972) terminology, to use and produce resources that suit their own local needs and beliefs require real, hands-on opportunities of local and personal adaptation. Critical literacy actions to this effect should be expressions of, or empower users to, local production and use of information that is representative of and useful for the contextual preconditions, information needs and knowledge beliefs of the user him/herself (e.g. Kapitzke, 2003a, b; Johansson and Limberg, 2017).

Both examples in Figure 2 illustrate in various ways how generic, standardised temporal measurements and values might disadvantage and misrepresent data subjects, whether individual persons or collective entities. Informed and empowered critical literacy approaches towards services and applications that offer visualisations with generic temporal representations require, firstly, that users are able to identify the unfair or misrepresentative effect, a challenge that is likely to vary in difficulty depending on the topic. Awareness of how a credit score is unfavourably affected by personal financial setbacks during the last year is probably more easily acknowledged than insights into how standardised medical recommendations may differ in general and from personal physical values in specific. Secondly, and once the first condition is achieved, it also requires that users know how, and have the possibilities, to adapt and adjust such representations of data from "above" or the "outside" so as to change the conditions in their own favour and own interests.

Turning to corresponding critical design-oriented suggestions of methods for addressing problems of undue reliance on and reproduction of standardised temporal measurements and values, van Dijk et al. (2016) also suggest possibilities to adapt and personalise the same. In the local community example of the grocery store (Figure 2B), the contextualised adaptation through local time data resulted in visualisations of community relevance, facilitated by the existence of suitable, simple, free visualisation techniques. The simplicity of a heatmap visualisation also has the added benefit of making it cognitively available to and useful for larger portions of the concerned population. However, applications and services in areas of finance and health are often commercial and proprietary, which might make the user more vulnerable to default, standardised temporal representations. Critical design strategies as discussed by Bardzell and Bardzell (2013) also seem less explicit or attuned to such "radical" solutions that would allow users to adjust and redesign for themselves. A greater emphasis on problematising ("speculative") rather than declarative ("truth-claiming") design approaches as mentioned (Bardzell and Bardzell, 2013) could, perhaps, decrease the need for individual users to adapt or adjust the given representation in the first place, yet the approach does not fall into the active empowerment goals of radical literacy.

\section{Counter}

The third dimension draws attention to more radical literacy approaches aiming to Counter and oppose externally defined and promoted representations that impose upon individuals and groups; it emphasises needs of individuals and groups to create alternatives to dominant, pre-existing, limiting, discriminatory or oppressive representations as expressions of power and control.

Oppressive representational bias is particularly interesting not least because we find here perhaps the most elaborate merger of critical literacy approaches with critical design strategies. Exercising critical - or radical - literacy in this context almost necessitates activities whereby the temporal (or other) representation in itself becomes the direct object of opposition through the creation of an alternative and/or transformed representation. The Indigenous community's alternative map in Figure 3A is part of the reactionary movement of counter-mapping (Peluso, 1995) (also known as "critical cartography" or "mapping back"), 
presenting a counter approach to representational privilege based on longstanding power structures. By allowing, in particular, Indigenous groups, people with functional variations and residents in disadvantaged areas, possibilities and tools to redraw maps as inscribed by colonialists, municipalities or more privileged residents and placing these in opposition to the formally accepted representations, the legitimacy of formerly non-problematised maps are questioned and challenged. In the process, the alternative views and voices become heard, and the marginalised may learn about themselves and thereby become empowered.

The examples in Figure 3 also illustrate that broad accessibility to and adjustability of visualisation tools and temporal representations are central for the empowerment of marginalised and oppressed groups and individuals. A certain mitigating effect might be achieved by adding multiple and multicultural pre-existing temporal representations to visualisations. Subjective experiences of time, however, cannot be so easily accommodated by pre-existing models and visualisation tools and will instead require opportunities for users' own representational adaptations and designs. It follows from this that empowerment of individuals and groups in this sense requires not only visualisation designs that allow adaptations of the general or standard temporal representation offered; means to become truly empowered require that users are able to create visualisations that express other temporal conceptions, experiences and regimes - whether they are of a personal character or part of a shared social system of a group or culture. As illustrated by the examples in Figure 3, counter-mapping often occurs in low-tech formats, which shows two important things. Firstly: sophisticated and adapted or versatile digital visualisation tools are not available to many of these groups. Secondly: and somewhat counterintuitive to the aforementioned, it shows that the medium may not be so important, it is the potential to question and challenge dominant representations by reshaping them, which enables the enactment of transformative empowerment. Progress in this area would be likely to support the alternative temporal needs in digital humanities research as well (c.f. Drucker, 2014, 2020).

In previous research, discrimination and power abuse through similar representational forces have mostly focussed on cartographic representation and Indigenous cultures. However, with the increasing digitisation, automation and complexity of media, services and governing institutions, in which representations of time are deeply embedded and instrumental in valorising, judgment-making and allocation of values and resources, the problem complex is likely to structurally and negatively affect ethical rights and power distribution concerning resources for also other types of groups and individuals in today's society. As in counter-mapping, visualisation research and design might fruitfully look to and engage minorities, other cultures and individual non-professionals to find needs and views on how alternative temporal representations might look and be produced to further other needs. To counter temporal power structures, tools need to be free, open and accessible (low thresholds) and the opposing voices need to be given platforms and be invited to participate on their own terms. Future digital data visualisation tools may, however, more easily allow for free alterations of time scales and other temporal representations.

\section{Cross}

The fourth and final critical literacy dimension encourages users and designers to Cross beyond the representational boundaries of the existing, conventional and familiar; it draws attention to the risks of forcing data into inadequate representational structures and to how it sometimes may be necessary to seek out entirely innovative ways of representing new or particularly complex phenomena.

The need to cross beyond current representations and thereby associated limits of thinking and reasoning (c.f. Hutchins, 1995) seems particularly urgent to address in light of recent and ongoing large-scale, global events and crises (c.f. e.g. Sampson, 2020). We find
Making time/ breaking time 
JD

78,1

today a number of critical, urgent issues, that researchers, theorists and political activists struggle to express and explain, and where it is apparent that dominant, long-established temporal representational limitations exist and may block possibilities to express and communicate about topics that involve, for the average person, thus far largely unfamiliar temporal scales, concepts and experiences.

In cases of this type, both the visualisation and its users need to cross beyond what is common, known, established and familiar to not impossibly constrain the possibilities to reflect, analyse and interpret to necessary extents. Critical aspects of this type seem largely overlooked in critical and radical literacy, but the challenge involved relates to topics of "deep uncertainty": “. . . uncertainty which we cannot quantify, which we cannot quantify given our available resources, or whose quantification is on balance undesirable" as discussed in visualisation for decision-making (Levontin et al., 2020) but also in relation to astronomy, geology, biology, evolution and other sciences that deal with very large scale or very smallscale temporal phenomena that are beyond the ordinary human perceptual experience and/or existing representational structures.

Designers of visualisations trying to communicate the interconnection between processes occurring at very different temporal scales face a particular problem. Slow changes occurring on large time scales, such as typical speciation time in mammals or the formation of fossil fuels, usually take hundreds of thousands or millions of years, whereas the current depletion of biological diversity due to human activity or the release of carbon dioxide to the atmosphere due to fossil fuel consumption is several orders of magnitude faster. This means that in the time scale relevant to humans, events occurring slowly over millions of years usually appear static unless they are carefully measured. An understanding of this requires what Bjørnerud (2018) calls "time literacy" including a "poly-temporal worldview" to acknowledge the overlapping rates of change, especially the imperceptible slow changes rooted in deep geological history. Possible design alternatives to resolve this issue are again suggested in terms of multiple representations (e.g. Ainsworth, 2008), reminiscent of the perspective-changing recommendations in critical design (Bardzell and Bardzell, 2013). Another approach is to provide users with interactive control over the presented information (e.g. Johansson, 2012; Stenlund and Tibell, 2019), and a third recognises that far from all visualisations are designed well enough to work as "stand-alone" tools for investigation and interpretation and that a supporting context (e.g. scaffolding resources such as text guides or instructors) is therefore needed to enable users to access the desired and available information in intended ways.

The final and perhaps utmost implication of the critical/radical literacy perspective here is to altogether abandon perceived limited and limiting existing representational possibilities in data visualisation for certain data or topics and instead "cross over" to other representational possibilities, such as an infographic. Of Bardzell and Bardzell's (2013) critical design suggestions, the one that seems to come closest to this dimension is that of a dialogical approach - although it speaks more about the design process than a particular, recommended, design form. In cases such as these, researchers and designers are even reported to look to the abstract and surrealist artworks of e.g. Rothko and Picasso for inspiration (Levontin et al., 2020), yet thus far, there are few examples of this sort of real-life visualisation applications outside, possibly, experiments and installations. The difficulties should not, however, as the authors argue, be used as an excuse for neglecting these aspects in visualisation design and development (Levontin et al., 2020).

\section{Conclusions}

The grounding of this conceptual discussion of temporal representations in a sociotechnical perspective means that we start from the view that they are both shaped by and shaping social, cultural, perceptions of, and actions based on, "time". That is, we see these 
representations as conducive to the making of time. Adding to this the critical and normative theories, methods and ideals of critical literacy, radical literacy and critical design, we also arrive at critical and ethical requests for responsible action and user empowerment through critical design strategies and appropriate literacy approaches. These should assist in users' possibilities to detect, change and oppose existing bias and disadvantageous political effects of these representations. In other words, they should function as critically appropriate and responsible ways of breaking time. From this position, we presented four types of political effects of temporal bias in visualisations expressed as limitation of view, disregard of variation, oppression of social groups, and misrepresentation of topic, and we suggested that appropriate critical and radical literacy approaches require users and designers to critique, contextualise, counter and cross beyond expressions of the same. Supporting critical design strategies involve the inclusion of multiple data sets and representations; broad access to flexible tools; and inclusive participation of marginalised groups.

In continuation, we conclude that temporally structured data visualisations can express and affect numerous and far-reaching political consequences. The implications raise issues of high and shared concern for library and information science, education, educational research, visualisation research and design and society at large. It is urgent to advance research and theory in the area, for which both visualisation design and critical/radical literacy research ought to conduct more situated user studies to uncover deeper knowledge of values and politics enacted through the design and use of temporal representations in visualisation.

From our discussion, we also conclude that critical and radical literacy are well suited to support and complement each other from the ethical perspectives of critical and responsible approaches and strategies of designers and users. The critical and radical literacy dimensions of the " $4 \mathrm{C}$ model" presented here can, in a further sense, also be seen to imply something of a hierarchy, ranging from a more passive "critiquing" approach of the critical literacy tradition whereby limitations of representations can and should become identified and questioned both for their own sake and as a prerequisite to the other dimensions; to the "contextualising" approach in which standard or arbitrary temporal scales and intervals should not only be questioned but preferably also adapted to suit local and subjective perspectives; to the more radical, transformative approaches of "countering", denoting aims to actively refute, rewrite and replace existing oppressive or excluding norms and effects of dominant representations; and the final "crossing" approaches that underline also the need to recognise situations for which the use of conceivable representations in the data visualisation register are inadequate to the point of being undesirable on the whole, and therefore more suitably approached through other representational means altogether, including infographics, text and similar resources. Integrating the strengths of these two perspectives - the critical and the radical provides a well-rounded approach to problems of bias and politics, as well as other similar types of social construction, use and interaction with complex information resources.

Placing emphasis on the third, critical design, perspective applied here, the discussion also leads us to conclude that mainstream data visualisation research and design should benefit by extending, through the application of critical design principles, beyond the current limited focus on multiple representations, towards (also) the potentials of critical design in a broader sense. The time is ready, for example, for users and the public at large to be allowed access to the fuzzy, messy, complicated real-life aspects of data, of visual representation and analysis. More and more researchers argue for the benefits of embracing and including a visual representation of a complex or unresolved character, such as uncertainty. Bonneau et al. (2014, pp. 10-11) argue that a "... systematic omission ... commands fundamental research within the visualization community to address, integrate, and expect uncertainty information." In a similar vein, McInerny et al. tie the entire trustworthiness of visualisations to the degree to which challenges of the scientific process and the production of visualisations themselves can be included and transparent (McInerny et al., 2014, p. 155; c.f. also Gschwandtner et al., 2016).

Making time/ breaking time 
JD

78,1

The above includes in particular investigating possibilities of including multiple data sets; openly displaying the messy stages of the entire scientific (or otherwise situated) data visualisation process; and allowing members of the public and marginalised groups the opportunity to participate in production and evaluation of temporal representations. Such approaches are described by Johansson (2012) as a combination of "enunciative" and "evocative" qualities of visualisation and propagated by Ynnerman et al. (2018) in a portmanteau of exploration and explanation as "exploranation". The views underline the critical in critical design, of reflexive awareness of visualisation itself as an actor; of a dialogical methodology; a problematising rather than declarative approach; and perspectivechanging accounts of data, visualisation, science and research itself. All of which contribute to raise the cultural competence among users (Bardzell and Bardzell, 2013). What seems altogether lacking, thus far, however, are equivalents in critical design to the radical approaches of radical literacy, for which the design process needs to not only provide solutions to users but also invite them into processes, and sometimes allowing them to take over in full. It is desirable thus to reshift visualisation focus in general from product to process and emphasising more explicitly in both talk about, design and uses of visualisations their values as tools for discovery, question formulation and critical reasoning.

This discussion also leads us to argue that, contrary to certain assumptions, there may be more that unites needs and challenges concerning the visual representation of time and temporality in the empirical sciences and humanities than there is that separates them. Researchers and practitioners in both areas seek ways to visually represent temporally related knowledge and experience that is well-founded, well established or truly exploratory and ground-breaking, but that lacks data of precise, unidirectional, timeline applicable nature or whose very core characteristics do not conform with the alleged stronghold of the so-called "Kantian" timeline (c.f. Critchley, 2016). The critiques are not only founded upon but may also contribute to unnecessarily perpetuating dichotomic, strongly conflicting notions of time in "empirical science" on the one hand, and "humanities" on the other, which are not representative of actual visualisation challenges and desires in either area.

The powerful and politically potent representations of time and temporality can be expressed in various modes and modalities (numbers, texts, visuals, sound and haptics) but the politics of time in a broad sense cuts across modes and therefore needs to be recognised and theorised in comprehensive ways. We need continued research and education in specific literacies such as data literacy and visual literacy, to provide, as Markham (2020) notes, the basic knowledge needed to orient oneself and know when a need to take critical action arises. Adding on top of, or in addition to, such competencies, a more generic critical mindset towards potential bias and politics of temporal representation brings us, however, to literacy dimensions of critical and radical character, as "a way of being and doing" in all contexts and activities of interaction with information resources as described by Vasquez et al. (2019). Broadening the literacy approach to outside of formal learning contexts to everyday interactions with information resources with various representations of time leads to critical questions concerning how critical approaches and actions on behalf of users can be supported through conscious critical design strategies. As we have suggested, this can be foremost supported through providing tools for and promoting access to and incentives for engaging in processes that support critical insights, dialogue and choice, setting up a network for democratising and possibly also a representation-innovating plurality of voices and perspectives.

\section{References}

Aigner, W., Miksch, S., Schumann, H. and Tominski, C. (2011), Visualization of Time-Oriented Data, Springer, London, doi: 10.1007/978-0-85729-079-3. 
Ainsworth, S. (2008), "How do animations influence learning", in Current Perspectives on Cognition, Learning, and Instruction: Recent Innovations in Educational Technology that Facilitate Student Learning, pp. 37-67.

Andersen, J. (2006), "The public sphere and discursive activities: information literacy as sociopolitical skills", Journal of Documentation, Vol. 62 No. 2, pp. 213-228.

Ayalasomayajula, V. (2016), "Visualizing time series data: 7 types of temporal visualizations", Humans of Data, 1 November, available at: https:/humansofdata.atlan.com/2016/11/ visualizing-time-series-data/ (accessed 27 April 2021).

Bardzell, J. and Bardzell, S. (2013), "What is 'critical' about critical design?”, CHI 2013, April 27-May 2, 2013, Paris.

Beaulieu, A. (2002), "Images are not the (only) truth: brain mapping, visual knowledge, and iconoclasm”, Science, Technology and Human Values, Vol. 27 No. 1, pp. 53-86, doi: 10.1177/ 016224390202700103.

Beer, D. and Burrows, R. (2013), "Popular culture, digital archives and the new social life of data", Theory, Culture and Society, Vol. 30 No. 4, pp. 47-71.

Bendoly, E. (2016), "Fit, bias, and enacted sensemaking in data visualization: frameworks for continuous development in operations and supply chain management analytics", Journal of Business Logistics, Vol. 37, pp. 6-17, doi: 10.1111/jbl.12113.

Bjørnerud, M. (2018), Timefulness: How Thinking like a Geologist Can Help Save the World, Princeton University Press, Princeton.

Blackwell, A. (2011), "Visual representation”, in Soegaard, M. and Dam, R.F. (Eds), Encyclopedia of Human-Computer Interaction, The Interaction-Design Foundation, Aarhus, available at: http:// www.interaction-design.org/encyclopedia/visual_representation.html (accessed 27 April 2021).

Bonneau, G.P., Hege, H.-C., Johnson, C.R., Oliveira, M.M., Potter, K., Rheingans, P. and Schultz, T. (2014), "Overview and state-of-the-art of uncertainty visualization", in Hansen, C., Chen, M., Johnson, C., Kaufman, A. and Hagen, H. (Eds), Scientific Visualization, Springer, London, doi: 10. 1007/978-1-4471-6497-5_1.

Bremer, N. (2015), "Traffic accidents: comparing the number of traffic accidents to weather, holidays, and daylight hour patterns", VisualCinnamon.com, available at: https:/www.visualcinnamon. com/portfolio/traffic-accidents (accessed 27 April 2021).

Brumberger, E. (2019), "Past, present, future: mapping the research in visual literacy", Journal of Visual Literacy, Vol. 38 No. 3, pp. 165-180.

Calzada Prado, J. and Marzal, M.Á. (2013), "Incorporating data literacy into information literacy programs: core competencies and contents", Libri, Vol. 63 No. 2, pp. 123-134.

Craig, J. and Guzmán, L. (2018), "Six propositions of a social theory of numeracy: interpreting an influential theory of literacy", Numeracy, Vol. 11 No. 2, Article 2, doi: 10.5038/1936-4660.11.2.2.

Critchley, S. (2016), “Times”, Parse, Vol. 4, available at: http://parsejournal.com/article/times/ (accessed 27 April 2021).

Dimara, E., Franconeri, S., Plaisant, C., Bezerianos, A. and Dragicevic, P. (2020), "A task-based taxonomy of cognitive biases for information visualization", IEEE Transactions on Visualization and Computer Graphics, Vol. 26 No. 2, pp. 1413-1432, doi: 10.1109/TVCG.2018. 2872577.

Drucker, J. (2014), Graphesis: Visual Forms of Knowledge Production, Harvard University Press, Cambridge, MA.

Drucker, J. (2020), Visualization and Interpretation: Humanistic Approaches to Display, MIT Press, Cambridge, MA and London.

Dunne, A. and Raby, F. (2001), Design Noir: The Secret Life of Electronic Objects, Birkhäuser, Basel.

Elkins, J. (Ed.) (2009), Visual Literacy, Routledge, New York, NY. 
JD

78,1

Eryaman, M.Y. (2010), "The public library as a space for democratic empowerment: Henry Giroux, radical democracy, and border pedagogy", in Leckie, G.J., Given, L.M. and Buschman, J.E. (Eds), Critical Theory for Library and Information Science: Exploring the Social from across the Disciplines, ABC-CLIO, LLC, Santa Barbara, pp. 131-141.

Eurostat (2020), "Road accident fatalities, EU-27", Eurostat, European Commission, available at: https://ec.europa.eu/eurostat/statistics-explained/index.php/Road_accident_fatalities_-statistics_by_type_of_vehicle (accessed 27 April 2021).

Freire, P. (2000/1972), Pedagogy of the Oppressed, Continuum, New York.

Friendly, M. (2008a), "A brief history of data visualization”, in Chen, C.-H., Härdle, W. and Unwin, A. (Eds), Handbook of Data Visualization, Springer-Verlag, Berlin and Heidelberg, pp. 15-56.

Friendly, M. (2008b), "The golden age of statistical graphics", Statistical Science, Vol. 23 No. 4, pp. 502-535.

Gschwandtner, B., Bögl, M., Federico, P. and Miksch, S. (2016), "Visual encodings of temporal uncertainty: a comparative user study", IEEE Transactions on Visualization and Computer Graphics, Vol. 22 No. 1, pp. 539-548.

Hess, D., Amir, S., Frickel, S., Kleinman, D.L., Moore, K. and Williams, L.D.A. (2017), "Structural inequality and the politics of science and technology", in Felt, U., Fouché, R., Miller, C.A. and Smith-Doerr, L. (Eds), The Handbook of Science and Technology Studies, MIT Press, Cambridge, MA, pp. 319-347.

Hsu, Y.-C., Dille, P., Cross, J., Dias, B., Sargent, R. and Nourbakshsh, I. (2017), “Community empowered air quality monitoring system”, CHI 2017, May 6-11, 2017, Denver, CO.

Hutchins, E. (1995), Cognition in the Wild, MIT Press, Cambridge, MA.

ICA Toria Supermarket (2020), "Customer frequency per weekday and hour”, Visualisation/Facebook post, ICA Toria Supermarket, Torsby.

Jewitt, C. and Kress, G.R. (Eds) (2003), Multimodal Literacy, Lang, New York.

Job One for Humanity (n.d.a.), "Earth's atmospheric $\mathrm{CO} 2$ concentration and temperature", available at: https://www.joboneforhumanity.org/the_4_most_critical_global_warming_deadlines_and_ tipping_points (accessed 27 April 2021).

Job One for Humanity (n.d.b.), "Planetary threshold line", available at: https://www. joboneforhumanity.org/the_4_most_critical_global_warming_deadlines_and_tipping_points (accessed 27 April 2021).

Johansson, V. (2012), A Time and Place for Everything? Social Visualisation Tools and Critical Literacies, The University of Borås, Borås, available at: http://urn.kb.se/resolve?urn=urn:nbn:se:hb:diva-3638.

Johansson, V. and Limberg, L. (2017), "Seeking critical literacies in information practices: reconceptualising critical literacy as situated and tool-mediated enactments of meaning", Information Research, Vol. 22 No. 1, available at: http:/InformationR.net/ir/22-1/colis/ colis1611.html.

Johnson, G.M. (2020a), "Algorithmic bias: on the implicit biases of social technology", Synthese, Vol. 198, pp. 9941-9961, doi: 10.1007/s11229-020-02696-y.

Johnson, G.M. (2020b), “The structure of bias”, Mind, Vol. 129 No. 5162, pp. 1193-1236, doi: 10.1093/ mind/fzaa011.

Johnson, C.R. and Sanderson, A.R. (2003), “A next step: visualizing errors and uncertainty”, IEEE Computer Graphics and Applications, Vol. 23 No. 5, doi: 10.1109/MCG.2003.1231171.

Joyce, K. (2005), “Appealing images: magnetic resonance imaging and the production of authoritative knowledge", Social Studies of Science, Vol. 35 No. 3, pp. 437-462.

Kapitzke, C. (2003a), "Information literacy: a positivist epistemology and a politics of outformation", Education Theory, Vol. 53 No. 1, pp. 37-53.

Kapitzke, C. (2003b), "Information literacy: a review and poststructural critique", Australian Journal of Language and Literacy, Vol. 26 No. 1, pp. 53-66. 
Kennedy, H., Hill, R.L., Aiello, G. and Allen, W. (2016), "The work that visualisation conventions do", Information, Communication and Society, Vol. 19 No. 6, pp. 715-735.

Kocinkova, L. and Hale, C. (n.d.), "Visualizing TFL accident data (2015)", available at: https://lucyia. github.io/tfl-accidents/ (accessed 27 April 2021).

Koltay, T. (2015), "Data literacy: in search of a name and identity", Journal of Documentation, Vol. 71 No. 2, pp. 401-415.

Lankshear, C. and McLaren, P. (1993), Critical Literacy: Politics, Praxis, and the Postmodern, University of New York Press, State.

Latour, B. and Woolgar, S. (1986/1979), Laboratory Life: The Social Construction of Scientific Facts, Princeton University Press, Princeton, NJ.

Lee, H.-S., Liu, O.L., Price, C.A. and Kendall, A.L.M. (2011), "College students' temporal-magnitude recognition ability associated with durations of scientific changes", Journal of Research in Science Teaching, Vol. 48 No. 3, pp. 317-335, doi: 10.1002/tea.20401.

Leftwich, A. (1983), Redefining Politics: People, Resources and Power, Methuen, London.

Lemke, J.L. (2000), “Across the scales of time: artifacts, activities, and meanings in ecosocial systems", Mind, Culture, and Activity, Vol. 7 No. 4, pp. 273-290, doi: 10.1207/S15327884MCA0704_03.

Levontin, P., Walton, J.L., Kleineberg, J., Barons, M., French, S., Aufegger, L., McBride, M., Smith, J.Q., Barons, E. and Houssineau, J. (2020), "Visualising uncertainty: a short introduction", AU4DM, London, available at: http://kleineberg.co.uk/wp-content/uploads/2019/12/VUI_221219.pdf.

Lurie, N.H. and Mason, C.H. (2007), "Visual representation: implications for decision making", Journal of Marketing, Vol. 71 No. 1, pp. 160-177, doi: 10.1509/jmkg.71.1.160.

Mackenzie, K., Siabato, W., Reitsma, F. and Claramunt, C. (2017), "Spatio-temporal visualisation and data exploration of traditional ecological knowledge/indigenous knowledge", Conservation and Society, Vol. 15 No. 1, pp. 41-58.

Mandinach, E.B. and Gummer, E.S. (2013), "A systemic view of implementing data literacy in educator preparation”, Educational Researcher, Vol. 42 No. 1, pp. 30-37.

Mapa da Aldeiaboa vista (n.d.), "Social cartography example", available at: notanatlas.org.

Markham, A. (2020), "Taking data literacy to the streets: critical pedagogy in the public sphere", Qualitative Inquiry, Vol. 26 No. 2, pp. 227-237.

McInerny, G.J., Chen, M., Freeman, R., Gavaghan, D., Meyer, M., Rowland, F., Spiegelhalter, D.J., Stefaner, M., Tessarolo, G. and Hortal, J. (2014), "Information visualisation for science and policy: engaging users and avoiding bias", Trends in Ecology and Evolution, Vol. 29 No. 3, pp. 148-157, doi: 10.1016/j.tree.2014.01.003.

Moreno, R. and Mayer, R. (2007), "Interactive multimodal learning environments", Educational Psychology Review, Vol. 19 No. 3, pp. 309-326, doi: 10.1007/s10648-007-9047-2.

Notanatlas.org (n.d.), "A new social cartography: defending traditional territories by mapping in the Amazon", in This Is Not an Atlas, available at: https://notanatlas.org/maps/a-new-socialcartography/ (accessed 27 April 2021).

Nygårds, K. (2020), “Totally unpredictable”, available at: https://www.facebook.com/karin.nygards/ posts/10160269900415620 (accessed 27 April 2021).

Peluso, N.L. (1995), "Whose woods are these? Counter-mapping forest territories in Kalimantan, Indonesia”, Antipode, Vol. 4 No. 27, pp. 383-406, doi: 10.1111/j.1467-8330.1995.tb00286.x.

Riedler, M. and Eryaman, M.Y. (2010), "Transformative library pedagogy and community-based libraries: a Freirean perspective", in Leckie, G.J., Given, L.M. and Buschman, J.E. (Eds), Critical Theory for Library and Information Science: Exploring the Social from across the Disciplines, ABC-CLIO, LLC, Santa Barbara, pp. 89-99.

Sampson, K. (2020), "Conceptions of temporality: reconsidering time in an age of impending emergency", Theoria, Vol. 86 No. 6, pp. 769-782.

Making time/ breaking time

\section{$+$}


JD

78,1

Stenlund, J. (2019), Travelling through Time: Students' Interpretation of Evolutionary Time in Dynamic Visualizations, Linköping University Press, Linköping, doi: 10.3384/lic.diva-154619.

Stenlund, J. and Tibell, L.A.E. (2019), "Visualizing macroevolutionary timescales: students' comprehension of different temporal representations in an animation", Evolution: Education and Outreach, Vol. 12 No. 1, doi: 10.1186/s12052-019-0099-9.

Tewell, E. (2015), "A decade of critical information literacy: a review of the literature", Communications in Information Literacy, Vol. 9 No. 1, pp. 24-43, doi: 10.15760/comminfolit.2015.9.1.174.

Tuominen, K., Savolainen, R. and Talja, S. (2005), "Information literacy as a sociotechnical practice", Library Quarterly, Vol. 75 No. 3, pp. 329-345.

van Dijk, E.K., IJsselsteijn, W. and Westerink, J. (2016), Deceptive visualizations and user bias: a case for personalization and ambiguity in PI visualizations, Proceedings of the 2016 ACM International Joint Conference on Pervasive and Ubiquitous Computing: Adjunct (UbiComp '16), Association for Computing Machinery, New York, NY, pp. 588-593, doi: 10.1145/2968219. 2968326.

Vasquez, V.M., Janks, H. and Comber, B. (2019), "Critical literacy as a way of being and doing", Language Arts, Vol. 96 No. 5, pp. 300-311.

Whitworth, A. (2014), Radical Information Literacy: Reclaiming the Political Heart of the IL Movement, Chandos, Oxford.

Ynnerman, A., Löwgren, J. and Tibell, L. (2018), "Exploranation: a new science communication paradigm", IEEE Computer Graphics and Applications, Vol. 38 No. 3, pp. 13-20, doi: 10.1109/ MCG.2018.032421649.

\begin{abstract}
About the authors
Veronica Johansson is a Senior Lecturer in Library and Information Science at the Swedish School of Library and Information Science (SSLIS), University of Borås, Sweden and Guest Lecturer in visual learning and communication at the Department of Science and Technology (ITN), Linköping University, Sweden. Her research and teaching are focussed on data as applied in visualisations and AI technology and related consequences from the perspectives of user interaction; critical literacy; privacy and ethics; and critical and value-sensitive design. Profile page: https://www.hb.se/en/research/research-portal/ researchers/VEJ/. Veronica Johansson is the corresponding author and can be contacted at: veronica. johansson@hb.se

Jörgen Stenlund is a Lecturer at the School of Science and Technology, Örebro University and a PhD Candidate at the Department of Science and Technology (ITN) and Department of Behavioural Sciences and Learning (IBL), Linköping University, Sweden. In his research, he has focussed on visual learning and communication in sciences education. Jörgen's dissertation focusses on visualisations of time and timescales in evolution. He is affiliated to the group Visual Learning and Communication at Linköping University. Profile page: https://www.oru.se/english/employee/jorgen_stenlund
\end{abstract}

For instructions on how to order reprints of this article, please visit our website:

www.emeraldgrouppublishing.com/licensing/reprints.htm

Or contact us for further details: permissions@emeraldinsight.com 